\title{
Aus dem Vorwort zur ersten Auflage.
}

Ohne daß es in meiner Absicht liegen konnte, mit den größeren selbständigen Kommentaren über das Strafgesetzbuch und über die Strafprozeßordnung in Konkurrenz zu treten, oder eine vollständige Sammlung der noch neben dem Strafgesetzbuche... geltenden Strafgesetze zu liefern, so sollte doch so viel Material geboten werden, um in der weitaus größten Mehrzahl der Fälle die Zurhandnahme noch anderer Bücher entbehrlich zu machen. Aber der Wunsch, recht viel zu geben, mußte seine natürliche Einschränkung in der Rücksicht finden, daß dem Buche nicht durch einen zu großen Umfang die handliche Form geraubt werden dürfe, welche für ein Vademecum des Kriminalisten, wic es hier geschaffen werden sollte, ganz unerläßlich erschien.

Marienwerder, im Mai 1879.

\section{A. Dalcke.}

\section{Vorwort zur 35. Auflage.}

Die 34. Auflage dieses Werkes erschien zwar erst im Frühjahr 1948. Es handelte sich dabei aber um einen Torso der schon 1943/44 bearbeiteten Neuauflage, die bei Kriegsende fertig ausgedruckt vorlag und deren Druckbogen z. T. bei dem Zusammenbruch verloren gingen. Im Gegensatz dazu stellt sich die 35. Auflage als vollständige Neubearbeitung dar.

Die neue Auflage stellte die Bearbeiter vor Probleme, wie sie bei den vorangegangenen Auflagen nicht aufgetaucht waren. Sie ergaben sich aus der seit $194 j$ eingetretenen erschreckenden Rechtszersplitterung in Deutschland. Die früheren Auflagen brachten im wesentlichen die in ganz Deutschland geltenden Reichsgesetze, an Gesetzen mit örtlich beschränktem Geltungsbereich dagegen nur einige Gesetze Preußens als des größten deutschen Landes. Seit 1945 aber weicht nicht nur das Recht der einzelnen Besatzungszonen, sondern auch das der Länder innerhalb der einzelnen Besatzungszonen voneinander ab. Und soweit förmliche Änderungen des am 8. 5. 1945 geltenden Rechts nicht erfolgt sind, bestehen mannigfache Zweifel über seine Weitergeltung. Welchen Text sollten unter diesen Umständen die Bearbeiter bringen, wenn sie einem möglichst großen Benutzerkreis dienen und dabei den Grundg€danken des Werkes im Rahmen des Möglichen beibehalten wollten, nämlich - wie der erste Herausgeber im Vorwort zur 1. Aufl. es ausgedrückt hatte - , jedem so viel Material zu bicten, ,um in der weitaus größten Mehrzahl der Fälle die Zurhandnahme noch anderer Bücher entbehrlich zu machen" ? Schwierigkeiten nach dieser Richtung bietet in erster Linie die StPO., beträchtliche Schwierigkeiten ergeben sich bei den Nebengesetzen, aber auch für da s StGB. sind sie nicht gering. Der Weg, den seit 1945 die Herausgeber von Textausgaben z. T. gewählt haben, lediglich den in einer bestimmten Zne oder gar in einem bestim.mten Land geltenden Text zu bieten, verbot sich bei Anlage und Umfang des Werkes von selbst. Auf der anderen Seite ist die vollständige 
Vorwort.

wörtliche Wiedergabe oder auch nur lückenlose Aufzählung aller in jedem Land seit 1945 erfolgten Änderungen, die die zahlreichen in dem Werk vereinigten reichsrechtlichen Vorschriften betreffen, ein Ding der Unmöglichkeit. Schon die Notwendigkeit, den Umfang des Werkes auf ein angemessenes $\mathrm{MaB}$ zu beschränken und die Übersichtlichkeit nicht zu gefährden, hätte dem entgegengestanden. Hinzu kommt, daß es sich oft nicht um förmliche Änderungen handelt, sondern da $B$ die Geltung der reichsrechtlichen Vorschriften nur mittelbar (einschränkend oder erweiternd) berührt wird. Die Bearbeiter haben sich daher zu folgender Lösung auf mittlerer Linie entschlossen:

1. Was das Strafgesetzbuch anlangt, so ist, um einen festen Ausgangspunkt $\mathrm{zu}$ haben und nicht einen willkürlich nach der privaten Auffassung des Bearbeiters zurechtgestutzten Text zu liefern, der Text - im Anschluß an die in Schleswig-Holstein 1946 erfolgte offiziöse Bekanntmachung - in der am 8. 5. 1945 geltenden Fassung unter Berücksichtigung der Streichungen, die die Kontrollratsgesetze Nr. 11 und 55 vornahmen, zugrunde gelegt. Zonen- und landesrechtliche Änderungen und Ergänzungen, wie z. B. bei §§ 120,132, 184, 218ff. StGB., sind bei kürzeren Texten wörtlich wiedergegeben, im übrigen durch entsprechende Hinweise berücksichtigt. Dabei ist allerdings hervorzuheben, daß das thüringische Strafrechtsanwendungsgesetz v. 1. 11. 1945 mit Rücksicht darauf, daß vollständige Ausgaben des Thüringischen StGB.-Textes vorliegen, nur erwähnt wurde, sowert es seinerseits später Änderungen erfahren hat.

In der Frage der Weitergeltung von Vorschriften, die in der Zeit nach dem 30. 1. 1933 reichsrechtlich geändert oder neu in das StGB. eingefügt worden sind, ohne nach dem 8. 5. 1945 förmlich geändert oder außer Kraft gesetzt zu scin, ist der Ausgangspunkt der Prüfung in West- und Ostdeutschland verschieden. Der Westen legt grundsätzlich den Rechtszustand v. 8. 5. 1945 zugrunde und erklärt nur spezifisch nationalsozialistisches Recht für unanwendbar (vgl. Art. II] des MilRegGes. Nr. 1). Über die Auswirkungen dieses Grundsatzes herrscht bei einer Reihe von Vorschriften Streit, wie etwa bei den $\S \S 49 \mathrm{a}, 175 \mathrm{f}, 239 \mathrm{a}, 240,253$, 330c StGB., § 20 RJGG. Die östliche Besatzungszone geht von der am 31. 1. 1933 geltenden Fassung aus und berücksichtigt spätere reichsrechtliche Änderungen nur, ,,scweit sie das Ergcbnis einer von nat.soz. Gedanken unal)hängig€n R€chtsєntwicklung sind" (so VO. der Prov. Sachsen v. 6. 2. 1946, VOBl. S. 306, ähnlich RdErl. des Präs. der Prov. Brandenburg v. 11. 10. 1945), VOBl. S. 81). Rechtsprechung und Schrifttum, die sich mit der Frage der Weitergeltung beschäftigen, sind jeweils in den Anmerkungen zu den einzelnen Vorschriften berücksichtigt. Der früher geltende Text ist dabei wörtlich oder inhaltlich wiedergegeben.

Für die Westzonen und Berlin ist darüber hinaus die Einwirkung der Ziff. 8 b der Allgemeinen Anweisung an Richter Nr. 1 (AAR. 1) ${ }^{1}$ ) auf die seit dem 30. 1. 1933 erfolgten Gesetzesänderungen zu prüfen. Die AAR. 1 ist unter A 4 abgedruckt und erläutert. Um auf die Notwendigkeit einer solchen Prüfung hinzuweisen, ist jeweils bei den einzelnen Vorschriften des StGB. vermerkt, ob sie nach $d \in \mathrm{m}$ 30. 1. 1933 geändert oder neu eingefügt wurden. Bei häufiger angcwendeten Vorschriften ist auch hier die frühere Fassung wörtlich oder inhaltlich angegeben. Daß Rechtsprechung und Schrifttum, die sich mit der Auswirkung der Ziff. $8 \mathrm{~b}$ befassen, jeweils bei der Erläuterung der einzelnen Vorschriften verwertet wurden, bedarf keiner Hervorhebung.

2. Was Gerichtsverfassungsgesetz und Strafprozeßordnung anlangt, so sind die zahlreichen Zweifelsfragen über die Weitergeltung der seit 1933

1) Für Berlin Ziff. 10 der Anw. v. 10.5. 1946. 
erfolgten Änderungen, die gerade auf diesem Gebiet mit Sicherheit hervorgetreten wären, in der Hauptsache dadurch vermieden worden, da $B$ in den einzelnen Besatzungszonen authentische Texte geschaffen wurden ${ }^{1}$ ). Das vorliegende Werk bringt die Textvarianten aller 4 Besatzungszonen. Der Bearbeiter ist dabei im allgemeinen in folgender Weise vorgegangen: Vorschriften, die überall in gleicher Fassung gelten, sind ohne besondere Bemerkung wiedergegeben. Weichen die Zonenfassungen voneinander ab, was meist nur bei den nach dem 30.1. 1933 geänderten Vorschriften der Fall ist, so ist grundsätzlich der vor dem 30.1.1933 geltende Text der StPO. an die Spitze gestellt und ersichtlich gemacht, inwieweit die übrigen Zonenfassungen abweichen. Die Abweichungen von dem am 8. 5. 1945 geltenden Text sind ebenfalls hervorgehoben. Dieses Verfahren liegt im Sinne des in den Westzonen z. Z. dem Bundestag vorliegenden Entwurfs eines Gesetzes zur Wiederherstellung der Rechtseinheit auf dem Gebiet der Gerichtsverfassung und des Verfahrensrechts in Zivil- und Strafsachen; er will unter Aufrechterhaltung solcher seit 1933 erfolgter Änderungen, die sich als eine nicht auf nat.-soz. Gedankergängen beruhende Weiterentwicklung des Rechts darstellen, grundsätzlich auf den am 30.1. 1933 bestehenden Rechtszustand zurückgreifen. Es wurde dabei, um den Umfang des Werkes nicht unnötig anschwellen zu lassen, ange-

1) a) In der LS-Zone das StGVG. 1946 und die StPO. 1946 (Strafrechtspflegeordnung 1946) mit EGStrPflO. 1946, wobei die Texte der einzelnen Länder geringfügig voneinander abweichen (vgl. für Bayern GVBl. Nrr. 8 vom 30. 3. 1946, für Württemberg-Baden RegBl. Nr. 9 vom 10. 5. 1946, für Hessen GVBl. Nr. 2 bis 6 vom 1. 3. 1946). Durch mehrere Länderratsgesetze wurde das GVG. in der Folgezeit 'rrgänzt. Dic Anordnungen der einzelnen Länder über die Wiedereinführung des Scnöffen- und Schwurgerichts und über die Besetzung der Strafkammern mit Schöffen (vgl. die Zusammenstellungen zu $\S 28,76,79$ GVG.) hatten $z$. T. weitgehende Verschiedenheiten in der Besetzung und sachlichen Zuständigkeit der Strafgerichte zur Folge:

1) in der brit. Zone die von der MilReg. erlassene Allg. Anweisung für Richter Nr. 2. Durch V'O. der brit. MilReg, voin 1. 10. 1945 (Amtsblatt S. j0) wurde diese Anweisung, die im wesentlichen dem in der US-Zone geltenden Recht entsprach, mit Gesetzeskraft ausgestattet. In der Folgezeit ist, zunăchst durch übereinstimmende VO.en der OJ,G.-Präsidenten, dann, nach Bildung des Zentraljustizamts, durch VO.en dieser Stelle der Text von GVG. und StPO. geändert worden, so daB jetzt erhebliche Unterschiede gegenüber dem Recht der amerik. Zone bestehen;

c) in der Sowjet-Zone die StPO. in der an 1. 1. 1949 geltenden Fassung, herausgegeben von der Deutschen Justizverwaltung der sowj. Besatzungszonc (Deutscher Zentralverlag Berlin O 17). Sic ist das Ergebnis einer Konferenz mit den Landesjustizverwaltungen, die die seit dem 30.1. 1933 erfolgten Inderungen auf ihre weitere Anwendbarkeit überprüfte, und besitzt keine Gesetzeskraft. Vgl. dazu Weiß $\mathrm{NJ} .48,215 \mathrm{tf}$.

d) In der franz. Zone ist auszugehen

a) bezüglich des Verfahrensrechts von dem am 8. 5. 1945 geltenden Recht,

B) bezüglich des Aufbaues, der Zuständigkeit und der Besetzung der Gerichte von dem GVG. i. (1. F. der Bek. vom 22. 4. 1924 unter Berücksichtigung der bis 30. 1. 1933 erfolgten Änderungen.

Die Lănder der franz. Zone erließen in den Jahren 1946 und 1947 entsprechende, inhaltlich ähnliche Rechtsanordnungen über die Gerichtsverfassung und das Verfahren (Rheinland-Pfalz: LandesVO. vom 11. 4. 1947 - VOBI. S. 155 - i. d. F. ller LandesVO. vom 1. 12. 1949 - GVBI. S. 599 -; Baden: Rechtsanordnung vom 9. 7. 1946 - ABl. S. 44 -; Württemberg-Hohenzollern: Rechtsanordnung vom 13. 8. 1946 - ABl. S. $230-$; bayr. Kreis Lindau: Rechtsanordnung vom 30. 6. 1947 - ABl. Nr. $50-$ ). (Vgl. DRZ. 46, 145, JR. 47, 152, Bloedhorn JR. 48, 211; DRZ. 48, 210). In Rheinland-Pfalz ist die StPO. durch Ges. vom 2. 9.1949 (GVBl. S. 373) in einer Reihe von Punkten geăndert, z. T. durch Angleichung an das Recht der brit. Zone (vgl. DRZ. 49, 495). 
strebt, den für die Darstellung der Varianten erforderlichen Raum möglichst klein zu halten. Da B bei dieser Textgestaltung der Benutzer der jeweiligen Zone ,,seinen“ Text weniger glatt lesen kann, als wenn er eine geschlossene Ausgabe des in dieser Zone geltenden Textes vor sich hat, versteht sich von selbst, aber das gilt für jede synoptische Darstellung, die auf beschränkten Raum angewiesen ist. Die Ersetzung des Wortes „Vorsitzer" durch „Vorsitzender" in der brit. Zone ist bei den einzelnen Vorschriften nicht besonders vermerkt.

Die in den Westzonen geltenden uneinheitlichen Vorschriften auf dem Gebiet der sachlichen Zuständigkeit der Strafgerichte und der Besetzung der Schöffengerichte, Strafkammern und Schwurgerichte, sowie der Auswahl der Schöffen und Geschworenen sind im Wortlaut wiedergegeben, soweit sie einheitlich innerhalb einer Zone gelten. Die voneinander abweichenden Vorschriften der Länder in der amerik. Zone sämtlich im Wortlaut aufzunehmen, fehlte der Raum; es ist deshalb nur die bayer. SchwurgerichtsVO. v. 14. 7. 1948 abgedruckt, während im übrigen eine inhaltliche Wiedergabe oder ein Hinweis auf die einschlägigen Vorschriften genügen mußte. Für die Ostzone ist das Gesetz über die Errichtung des Obersten Gerichtshofs vom 8.12. 1949 unter E II 3 aufgenommen.

3. Nebengesetze und strafprozessuale Verwaltungsvorschriften (Gnadenund Strafvollstreckungsordnung usw.) sind im allgemeinen in der beim Zusammenbrich geltenden Fassung unter Weglassung offensichtlich gegenstandslos gewordener Stellen wiedergegeben. Änderungen in den Zonen und Ländern (z. B. beim Reichspressegesetz, Reichsjagdgesetz, bei der Gnadenordnung usw.) sind im allgemeinen jeweils in Vorbemerkungen angeführt, z. T. auch bei der Erläuterung der einzelnen Bestimmungen verwertet, ohne da $B$ lückenlose Vollständigkeit angestrebt wäre ${ }^{1}$ ). Wiedergabe im Wortlaut erfolgte nur, soweit der beschränkte Raum es zulic $B$. Dagegen sind die Reichsgesetze ändernden und ergänzenden Vorschriften der früheren Doppelzone, die zum Teil auch in der franz. Zone eingeführt sind ( ..B. bei der Reichsabgabenordnung, der Straßenverkehrszulassungsordnung, dem Güterfernverkehrsgesetz, dem Patentgesetz), weil sie für ein großes

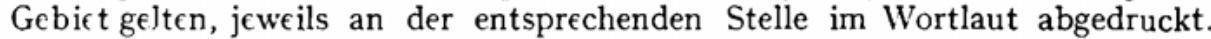
An Nebenrecht, das für größere Gebietsteile gilt, sind ferner das Wirtschaftsstrafgesetz der Doppelzone v. 26. 7. $1949^{2}$ ), die WirtschaftsstrafVO. der sowj. Zone v. 23. 9. 1948 nebst DurchfVO. und die bayer. Gnadenbekanntmachung v. 24. 7. 1947 vollständig aufgenommen.

Die Wiedergabe des früher allgemein geltenden Reichsgesetzes ist im übrigen nach Auffassung der Bearbeiter auch für die Gebietsteile, in denen durch neues Landesrccht das Reichsgesetz außer Kraft gesetzt ist, von Bedeutung, da dic bisherige Rechtsprechung mehr oder weniger für die Auslegung des neuen Rechts verwertbar sein wird. Dies gilt insbesondere da, wo - wie z. B. beim Reichsjagdgesetz - das neue Landesrecht sich weitgehend an das alte anschließt.

Bei Vorschriften, die auf nicht mehr vorhandene Stellen (z. B. die Reichsregierung, den Reichsminister der Justiz usw.) und Einrichtungen verweisen, ist der bisherige Text in Kleindruck wiedergegeben. Die jetzt zuständigen Stellen und die an die Stelle der früheren getretenen Einrichtungen ergeben sich aus dem

1) Eine Nachpriifung ter in der amerikanischen Zone erfolgten $\dddot{n}$ nderungen ermöglicht die ,Deutsche Gesetzesliste, System. Fundstellenverzeichnis aller Rechtsvorschriften und Rechtsänderungen seit dem 8. 5. 1945, Teil A", Biederstein-Verlag 1949, München.

2) Ihm entspricht in Rheinland-Pfalz das inhaltlich gleichlautende Gesetz v. 3. 9. 1949 (GVBI. S. 399); in clen übrigen Ländern der franz. Zone ist das Wirtschaftsstrafgesetz durch V). v. 24.1. 1950 (BGBl. S. 24) eingeführt. 
neuen Bundes-1) und Landesrecht, bzw. dem Bonner Grundgesetz (vgl. insbes. Art. 125, 129) und der Verfassung der Deutschen Demokratischen Republik (vgl. z. B. Art. 119, 124, 125). Besondere Hinweise darauf finden sich, wo nach Lage der Sache ein besonderer Anlaß dazu bestand.

Für die Nichtaufnahme einer Reihe von Gesetzen, die die früheren Auflagen enthielten, waren - soweit sie nicht offensichtlich gegenstandslos geworden sind - teils Gründe der räumlichen Beschränkung, teils der Gesichtspunkt maßgebend, da $B$ - wie $z$. B. bei dem Waffengesetz, dem Auslieferungsgesctz und dem (in den Westzonen ${ }^{2}$ ) neuerdings auch förmlich aufgehobenen) Devisengesetz - ihre Anwendung durch die deutschen Gerichte zur Zeit im Hintergrund steht, weil die Materie durch Besatzungsrecht geregelt ist, dessen Anwendung in der Hand der Besatżungsbehörden und -gerichte liegt. Aus Gründen der Raumersparnis ist für diese Auflage die Gewerbeordnung nicht aufgenommen worden. Es konnte dies um so mehr verantwortet werden, als die hauptsächlich in der amerik. Zone von der Besatzungsmacht angeordnete Beseitigung der Beschränkungen der Gewerbefreiheit ändernd in den Bestand der Vorschriften eingegriffen hat, die gesetzgeberische Fixierung der Änderungen im einzelnen aber noch aussteht. Nach erfolgter Klärung ist für künftige Auflagen die Wiederaufnahme der GewO. in Aussicht genommen. Inwieweit die Bearbeiter bei ihrer Auswahl das Richtige getroffen haben, bleibt abzuwarten. Für Hinweise aus der Praxis werden sie dankbar sein.

Besatzungsrecht ist insoweit im Wortlaut aufgenommen, als es a) den Tätigkeitsbereich der deutschen Gerichte abgrenzt (so das Kontrollratsges. Nr. 4 bzw. für Westdeutschland das Ges. Nr. 13 der Alliierten Hohen Kommission) oder b) das deutsche Recht förmlich ändert (wie $z$. B. die Kontrollratsgesetze Nr. 11 und 55) oder c) Tatbestände normiert, deren Úbertretung von den deutschen Gerichten, allgemein oder in einzelnen Zonen, abzuurteilen ist (wie z. B. die Kontrollratsgesetze Nr. 10 und 50). Besatzungsrecht, dessen Verletzung lediglich die Besatzungsbehörden und -gerichte ahnden, ist im allgemeinen nicht im Wortlaut aufgenommen, vielmehr an gehöriger Stelle darauf verwiesen.

Entscheidungen seit 1945, die in mehreren Zeitschriften veröffentlicht sind, sind, der früheren Ubung entsprechend, um Raum zu sparen, in der Regel nur mit einer Fundstelle zitiert. Sind sie in der ,Höchstrichterlichen Rechtsprechung in Strafsachen" (HESt.) veröffentlicht, so ist dieser Fundstelle meist der Vorzug gegeben worden. - Wenn auch die frühere reichsgerichtliche Rechtsprechung in weitestem Umfang ihre Bedeutung behalten hat und von den Revisionsjcrichten, die gebietsweise die Aufgaben des Reichsgerichts wahrnehmen, übernommen worden ist, so haben sie $z$. T. doch in einer Reihe von Fragen dem früheren höchsten Gericht die Gefolgschaft verweigert, ohne daß freilich bei der Vielzahl der Revisionsgerichte Einhelligkeit bestünde. Dies gilt $z$. B. für die Fragen der Bedeutung des Verbotsirrtums, für die Zulässigkeit der sog. konkreten Betrachtung bei der Einordnung der Tat in den Rahmen des $\S 1$ StGB., wenn „unbenannte“ Strafschärfungs- und -milderungsgründe den Strafrahmen bestimmen, für die Auslegung der Begriffe Unzuchttreiben in $\S 175 \mathrm{StGB}$. und unzüchtige Handlung in $\S 176$ Abs. 1 Ziff. 3 StGB., für die Frage, ob die in einem früheren Verfahrensstadium erfolgte Aussage des Zeugen, der in der Hauptver-

1) Wegen der neuen Verkündungsblätter, die an die Stelle der in früheren Gesetzen zenannten getreten sind, vgl. für die Westzonen $\$ 4$ des Ges. v. 30. 1. 1950 (BGBl. S. 23).

2) In der amerik. und brit. Zone bei der Neufassung des MilRegGes. Nr. 53 (vgl. Bundes«uziger N̈r. 2 v. 27. 9. 1949; Brit. Anitsblatt Nr. 39 v. 8. 10. 1949), die in der franz. Zone nit clem gleichen Wortlaut als VO. Nr. 235 (franz. Amtsblatt Nr. 305 v. 20.9. 1949) versidndet wurde. 
handlung vom Zeugnisverweigerungsrecht Gebrauch macht, durch Vernehmung der Verhörspersonen festgestellt und verwertet werden kann usw. Mit Rücksicht darauf sind, um zu erkennen, inwieweit die Gerichte sich der Rechtsprechung des RG. anschließen, nach dem 8. 5. 1945 ergangene Entscheidungen auch dann aufgeführt, wenn es sich um seit langem feststehende Grundsätze handelt und die neue Entscheidung sich lediglich anschließt.

Als das vorliegende Werk schon $z$. T. ausgedruckt war, hat die westdeutsche Bundesregierung dem Bundestag den obenerwähnten Entwurf eines Vereinheitlichungsgesetzes vorgelegt. Die Frage war, ob es unter diesen Umständen angezeigt sei, das Werk in der vorliegenden Form erscheinen zu lassen oder ob nicht zweckmäßiger mit dem Abschluß der Areitben bis zur Verabschiedung des Gesetzes innezuhalten sei. Die Entschließung mußte im Sinne der ersten Alternative ausfallen. Wenn auch die Notwendigkeit und Dringlichkeit des Gesetzes außer Frage steht, so ist doch nicht mit Sicherheit abzusehen, wie lange es noch bis zu seiner Verabschiedung dauert. Zwischen der Verabschiedung und dem Inkrafttreten des Gesetzes wird dann, wie anzunehmen, ein weiterer, nicht ganz unerheblicher Zeitraum liegen. Auch nach dem Inkrafttreten werden vermutlich für eine Úbergangszeit eine Reihe der bisherigen Vorschriften ihre Bedeutung behalten, etwa für die Frage der Anfechtbarkeit eines vor dem Inkrafttreten ergangenen Urteils. Schli $€$ Blich aber war zu berücksichtigen, daß die von der geplanten Änderung erfaßten Vorschriften nur einen verhältnismäßig kleinen Teil der in das Werk aufgenommenen Bestimmungen ausmachen dürften. Unter diesen Umständen erscheint es untunlich, das ohnedies durch drucktechnische Schwierigkeiten erheblich verzögerte Erscheinen des Werkes, nach dem in der Praxis eine lebhafte Nachfrage besteht, noch länger hinauszuschieben; sollte das Gesetz sehr bald nach Erscheinen des Werkes verabschiedet werden, so bleibt der Weg, es durch Lieferung eines Nachtrags für die Bezieher in vollem Umfang verwertbar zu erhalten.

Die Bearbeitung entspricht in den ersten Teilen dem Stand vom Spätherbst 1949. In den späteren Teilen sind neuere Gesetze und Entscheidungen soweit berücksichtigt, als es drucktechnisch noch möglich war (s. auch Nachträge S. 1197).

Es haben bearbeitet:

Dr. Fuhrmann: B I 6, II 1, 6-10, III 4-10, IV 3-9, V 5, VI, VII, VIII, C, D $1-3,5-7$, E I 4,5 , II, III $1-7$;

Dr. Schäfer: A $1-4$, B I $1-5$, II $2-5$, III $1-3$, IV $1-2$, V $1-4$, IX, D 4 , E I $1-3$, III $8-9$.

Die Bearbeiter würden sich freuen, wenn der Dalcke in dieser Form dem Praktiker wieder ein gleich brauchbares Rüstzeug für die tägliche Arbeit böte. wie es die früheren Auflagen darstellten. Für Hinweise auf Mängel und für Verbesserungsvorschläge werden wir dankbar sein.

Berlin und Frankfurt a. M., im Januar 1950.

Dr. E. Fuhrmann.

Dr. K. Schäfer. 